\title{
EARLY POSTOPERATIVE OUTCOMES IN PATIENTS WITH UTERINE CERVICAL CANCER
}

\author{
Mădălina Gavrilescu ${ }^{\bowtie}$, B. Filip, I. Huţanu, Mihaela Buna, N. Ioanid, \\ A. Pantazescu, V. Scripcariu \\ University of Medicine and Pharmacy „Gr.T. Popa” Iași \\ First Surgical Oncology Unit, Regional Institute of Oncology Iaşi, Romania
}

\begin{abstract}
EARLY POSTOPERATIVE OUTCOMES IN PATIENTS WITH UTERINE CERVICAL CANCER (Abstract): BACKGROUND: Uterine cervical cancer still presents high percentages of locally advanced tumors on diagnosis due to the lack of screening programs. The multidisciplinary approach in these cases requires neoadjuvant radio-chemotherapy followed in selected cases by surgery. The aim of this study was the assessment of early postoperative outcomes in a consecutive series of cervical cancer patients. METHODS: We performed a comparative analysis in terms of patients and tumor characteristics, type of surgery and postoperative complications on 83 patients with radical surgery divided in terms of neoadjuvant treatment protocol. Two groups were designed: group A, patients who underwent neoadjuvant therapy $(n=38 ; 45.78 \%)$ and group $\mathrm{B}$, patients operated without neoadjuvant therapy $(n=45$; $54.22 \%$ ). RESULTS: The patients mean age was 54.1 \pm 11.6 years old. Most cases on diagnosis were stage IIB (41 cases, $49.39 \%$ ) followed by stage IB (24 cases, 28.9\%). Neoadjuvant treatment was performed in 38 patients $(45.78 \%$ ) (stage IIA -1 patient, IIB -31 patients, IIIA -1 patient and IIIB - 5 patients), with a complete response in 16 (42.1\%). Most common intervention was type II radical hysterectomy with pelvic lymphadenectomy in 54 cases $(65 \%)$. There was no difference in terms of deep vein thrombosis, postoperative urinary and medical morbidity. Digestive complications were relatively higher in neoadjuvant group $(28.94 \%$ in group A vs. $6.66 \%$ in group $\mathrm{B}, P=0.008)$ with a longer hospitalization $(8.1 \pm 2.7$ days for group A vs. $6.6 \pm 1.6$ for group $\mathrm{B}, P=0.01)$. CONCLUSION: Radical hysterectomy is a relatively safe technique in terms of postoperative morbidity for patients with cervical cancer despite the use of neoadjuvant therapy. This provides the chance for cure in early stages and a local control for more advanced cases.
\end{abstract}

\section{KEY WORDS: UTERINE CERVICAL CANCER; NEOADJUVANT THERAPY; RADICAL HYSTERECTOMY}

\section{SHORT TITLE: Uterine cervical cancer}

HOW TO CITE: Gavrilescu M, Filip B, Huțanu I, Buna M, Ioanid N, Pantazescu A, Scripcariu V. Early postoperative outcomes in patients with uterine cervical cancer. Jurnalul de chirurgie (Iaşi). 2013; 9(3): 235-241. DOI: $10.7438 / 1584-9341-9-3-5$.

\section{INTRODUCTION}

The overall incidence rate of uterine cervical cancer in Europe is 10.6 per 100.000 , the incidence rates significantly differ, being lower in Western Europe where prevention programs are better developed and significantly higher in terms of incidence and mortality rates for Central and Eastern Europe, in close correlation to the intensity of organized screening [1].
In 1898, Ernst Wertheim from Vienna described the operation of radical hysterectomy which included removal of the parametrium and pelvic lymph nodes. Since that time, radical hysterectomy with pelvic lymphadenectomy has been performed with modifications in surgical technique as the major surgical treatment for early stage invasive cervical cancer [2]. The primary indication for radical hysterectomy with

Received date: 17.05 .2013

Accepted date: 20.06.2013

Correspondence to: Mădălina Gavrilescu MD

First Surgical Oncology Unit, Regional Institute of Oncology Iaşi

Str. General Henry Mathias Berthlot No. 2-4, 700483, Iaşi, Romania

Phone: 0040 (0) 0745514570

E-mail: madagavrilescu@yahoo.com 
pelvic lymphadenectomy is Stage I invasive cervical cancer. Patients with Stage IA2 or Stage IB1 cervical cancer have a significant risk of lymph nodal spread and should be treated by radical hysterectomy and pelvic lymphadenectomy [3]. Patients with Stage IB2 or Stage IIA cervical cancer are initially treated with chemoradiation or combined therapy in many institutions. However, selected patients with Stage IB2 or IIA cervical cancer may be treated with radical hysterectomy and pelvic lymphadenectomy [4]. The attempt to tailor the radicality according to tumor and patients characteristics seems to be one of the strategies to reduce the incidence of complications and their severity after surgical treatment of cervical cancer. The association of radiotherapy and radical surgery is generally recognized as an important risk factor for the occurrence of organ complications through vascular and fibrotic damage [5]. Complications of radical hysterectomy with pelvic lymphadenectomy includes: bladder dysfunction, bladder and ureteral injury, pelvic lymphocele, deep vein thrombosis or pulmonary embolism, blood vessel injury, prolonged ileus and wound infection [6].

The aim of this study was to evaluate the early postoperative outcomes after radical hysterectomy in terms of postoperative morbidity in a consecutive series of patients.

\section{MATERIAL AND METHOD}

We performed a retrospective study on a prospective collected database which included all the patients diagnosed with uterine cervical cancer in which surgery was performed between May 2012 and June 2013 in a tertiary oncologic centre. We included in the analysis all histological types of cervical tumors. In all patients preoperative staging was performed, including: histological confirmation through cervical biopsy, magnetic resonance imaging (MRI) of the pelvis, abdominal ultrasound. Based on preoperative staging and according to the recommendations of the multidisciplinary team, patients with tumors above stage FIGO IIA were considered candidates for neoadjuvant treatment.

This consisted in external beam therapy up to 50.4 Gy with or without concomitant chemotherapy; brachytherapy was not performed in patients included in this study. Post-induction therapy evaluation was performed after at least 4 weeks and included clinical and gynecological examination, full blood tests, MRI of pelvis, abdominal ultrasound.

All patients have undergone detailed comorbid assessment based on medical history and clinical examination (cardiac, pulmonary, hepatic, renal and metabolic diseases). Comorbidities of the patients were evaluated according to the Charlson scale [7] and general status of the patient according to the ASA scale. In all patients without distant metastasis surgery was performed and included radical hysterectomy with pelvic lymphadenectomy.

The extent of lymphadenectomy was dictated by the stage of the tumor and the results of the preoperative imaging studies. Radical hysterectomy was defined and classified according to the Surgery Committee of the Gynecological Cancer Group (GCG) of the European Organization for Research and Treatment of Cancer (EORTC) [8]:

- type I - simple hysterectomy,

- type II - modified radical hysterectomy (the uterus, paracervical tissues, and upper vagina $(1-2 \mathrm{~cm})$, the medial half of the parametria and proximal uterosacral ligaments are resected),

- type III - radical hysterectomy (en bloc removal of the uterus with the upper third of the vagina along with the paravaginal and paracervical tissues; the uterine vessels are ligated at their origin and the entire width of the parametria is resected bilaterally with removal of as much of the uterosacral ligaments as possible),

- type IV - extended radical hysterectomy (type III with three 
fourths of the vagina and paravaginal tissue are excised),

- type V hysterectomy (partial pelvic exenteration was not performed).

Systematic bilateral pelvic lymphadenectomy included: along the common iliac artery down to the femoral ring, including the presacral, both external, internal, and interiliac and the obturator nodes (at least to the level of the obturator nerve). In selected cases, a common iliac and aortic bifurcation lymphadenectomy was performed. Postoperative morbidity was defined as any complication that occurred within 30 days from surgery and was classified according to the Common Terminology Criteria for Adverse Events [9]. Postoperative pathological staging was performed using the FIGO and TNM classification [10].

Two groups were designed: group A, patients who underwent neoadjuvant therapy $(n=38 ; 45.78 \%)$ and group $\mathrm{B}$, patients operated without neoadjuvant therapy $(n=45$; $54.22 \%$ ). Demographic, clinical, biological, pathologic and operative data were included into a database and statistically analyzed.

\section{Statistical analysis}

Continuous data were expressed as median and interquartile range (IQR) or mean value with standard deviation. Categorical data were compared among the two groups using Fisher's exact test and continuous data using Mann-Whitney U test. We performed a comparative analysis between the characteristics of the patients in which neoadjuvant treatment was performed and those in which the induction therapy was not performed in terms of patients' characteristics, type of surgery and postoperative outcomes. Statistical analysis was performed using MedCalc v.9.2.0.1.

\section{RESULTS}

Between May 2012 and June 2013 in 83 patients diagnosed with cervical cancer, surgical intervention was performed. Table I shows the clinical characteristics of the patients. There was no difference in terms of age and Charlson comorbidity index. A detailed assessment of comorbid conditions on primary evaluation of the patients showed difference in prevalence of cardiac comorbidity. Most of the cases were squamocellular type tumors, less represented were the adenocarcinomas and the adenosquamous subtypes. Other types of tumors were clear cell carcinoma in 2 cases and neuroendocrine type in 1 case. Most frequent stage on diagnosis was FIGO stage IIB followed by the FIGO stage IB. Neoadjuvant treatment was performed in 38 cases and included only radiotherapy in 12 and concurrent chemo-radiotherapy in 26 cases.

The radiation dose ranged from 42.5 to $50.4 \mathrm{~Gy}$, all patients had undergone external beam radiotherapy, mean chemotherapy cycles number was 4. Chemotherapy regimen in patients who received CRT consisted of Cisplatin in 13 patients or Carboplatin and Taxol in 3 patients. There were 10 patients with locally advanced tumors (stage FIGO IIB) who were not considered suitable for neoadjuvant treatment. Most of all presented medical conditions, such as cardio-pulmonary chronic diseases in 4 cases, chronic renal disease 3 cases, liver conditions in 2 cases and diabetes in 1 case. Although they presented severe medical conditions, all of those patients were considered suitable for surgery due to the chronic hemorrhage from the tumor. By performing comparative analysis patients with neoadjuvant therapy presented before surgery lower values of hemoglobin and white blood cells.

All patients underwent a radical hysterectomy; the extent of surgery was performed accordingly to the tumoral stage. For stage IA and a small number of stage IB tumors, a type I radical hysterectomy was performed in 16 cases. In most cases was performed a type II radical hysterectomy (54 cases), more advanced cases received a radical type III hysterectomy (11 cases).

In all patients a radical pelvic lymphadenectomy was performed (Table II), the reason for performing a more radical 
lymphadenectomy (at the level of common iliac artery and aortic bifurcation) was the stage on re-evaluation after neoadjuvant treatment. In only 6 cases were found positive common iliac lymph nodes and on 2 cases positive aortic lymph nodes.

Based on the pathological examination 16 patients $(42.1 \%)$ presented a complete response on tumor and lymph nodes to neoadjuvant treatment.

Surprisingly, two patients presented a complete pathological response on tumor, but with positive lymph nodes.

There was no difference in term of resected number of lymph nodes.

Tumor down staging was obtained in 28 cases $(73.68 \%)$, and downsizing in 10 cases $(26.31 \%)$

Table I Patients' characteristics

\begin{tabular}{lcccc}
\hline & All patients & Group A & Group B & $P$ \\
\hline$n$ & 83 & 38 & 45 & \\
Age & $54.1 \pm 11.6$ & $53.5 \pm 12.5$ & $54.8 \pm 10.6$ & 0.60 \\
Histology: & & & & \\
Adenocarcinoma & $6(7 \%)$ & 0 & 6 & \\
Scuamocellular & $71(85 \%)$ & 36 & 1 & 0.09 \\
Adenosquamous & $3(4 \%)$ & 2 & 3 & \\
Other & $3(4 \%)$ & 0 & & - \\
Stage on diagnosis* & & & 11 & - \\
IA & $11(13 \%)$ & - & 24 & - \\
IB & $24(29 \%)$ & - & - & - \\
IIA & $1(1 \%)$ & 1 & - & - \\
IIB & $41(50 \%)$ & 31 & - & - \\
IIIA & $1(1 \%)$ & 1 & - & 0.65 \\
IIIB & $5(6 \%)$ & 5 & $3(2-3)$ & $\mathbf{0 . 0 0 0 1}$ \\
Radiotherapy & $12(32 \%)$ & 26 & 15 & 0.72 \\
Radio-chemotherapy & $26(68 \%)$ & $2(2-3)$ & 5 & 0.10 \\
Charlson score & $3(2-3)$ & 5 & 8 & 0.16 \\
Cardiac comorbidity & $20(36 \%)$ & 3 & 7 & 0.06 \\
Pulmonary comorbidity & $8(15 \%)$ & 2 & 7 & $\mathbf{0 . 0 4}$ \\
Liver disease & $10(18 \%)$ & 2 & $12.2 \pm 1.81$ & $\mathbf{0 . 0 0 1}$ \\
Diabetes & $9(16 \%)$ & $11.5 \pm 1.15$ & $7824 \pm 3871$ & \\
Renal disease & $8(15 \%)$ & $5129 \pm 1692$ & & \\
Hb & $11.9 \pm 1.57$ & & & \\
WBC & $6575 \pm 3330$ & & & - \\
\hline
\end{tabular}

Overall complications (as shown in Table III) rate was $40.9 \%$ (34 patients) and there was no difference between patients in which induction therapy was performed (20 cases) and patients only with surgical treatment (14 cases, $P=0.07)$. The most frequent adverse events were urologic complications as follow: bladder dysfunction in 12 cases, bladder injury in 8 cases and ureteral injury in 5 cases. There were no vesico-vaginal fistulas. All cases of bladder injury were identified intraoperatively and underwent primary repair during surgery with a $100 \%$ success rate. Four of the 5 ureteral injuries were recognized intraoperatively and underwent primary suture or anastomosis with a ureteral catheter inserted. One case underwent a cystocopic retrograde ureteral catheter insertion. Digestive complications occurred in 14 cases and were more frequent in patients with neoadjuvant therapy. The most 
common manifestation was prolonged ileus in 13 cases; one case developed a rectal perforation on the fifth postoperative day with a pelvic abscess which required surgical reintervention. Although the incidence of venous thrombosis was higher in patients with induction therapy, it was not statistically significant. There was no difference in terms of postoperative stay between the two groups.

Table II Surgical intervention and pathological examination

\begin{tabular}{lcccc}
\hline & Overall $(n=83)$ & Group A $(n=38)$ & Group B $(n=45)$ & $P$ \\
\hline Type I & $16(20 \%)$ & 3 & 13 & $\mathbf{0 . 0 2}$ \\
Type II & $54(67 \%)$ & 25 & 29 & 0.99 \\
Type III & $11(13 \%)$ & 8 & 3 & 0.10 \\
Pelvic lymphadenectomy & $83(100 \%)$ & 38 & 45 & - \\
Common iliac & $40(93 \%)$ & 17 & 23 & 0.66 \\
Aortic lymphadenectomy & $3(7 \%)$ & 3 & 0 & \\
Pathological stage & & & & \\
O (CR) & $16(19 \%)$ & 16 & 9 & 16 \\
IA & $14(17 \%)$ & 5 & 0 & - \\
IB & $27(33 \%)$ & 11 & 17 & \\
IIA & $2(2 \%)$ & 2 & & \\
IIB & $20(24 \%)$ & 3 & $16.6 \pm 9.9$ & 0.12 \\
III & $4(5 \%)$ & 2 & & \\
Resected lymph nodes $(n)$ & $18.2 \pm 9.5$ & $19.9 \pm 8.9$ & & \\
\hline
\end{tabular}

CR complete response

Table III Outcomes after surgery

\begin{tabular}{lcccc}
\hline & Overall $(n=83)$ & Group A $(n=38)$ & Group B $(n=45)$ & $P$ \\
\hline Overall morbidity & $34(41 \%)$ & 20 & 14 & 0.07 \\
Urinary complications & $19(23 \%)$ & 11 & 8 & 0.29 \\
Digestive complications & $14(17 \%)$ & 11 & 3 & $\mathbf{0 . 0 0 8}$ \\
Venous thrombosis & $5(6 \%)$ & 4 & 1 & 0.17 \\
Medical complications & $10(12 \%)$ & 6 & 4 & 0.50 \\
Mean hospital stay & $7.3 \pm 2.3$ & $8.1 \pm 2.7$ & $6.6 \pm 1.6$ & $\mathbf{0 . 0 1}$ \\
\hline
\end{tabular}

\section{DISCUSSION}

Cancer of the uterine cervix is staged using a clinical, rather than a surgical staging system, which is the approach used for other gynecologic tumors. In countries that do not have access to cervical cancer screening and prevention programs, cervical cancer remains the second most common type of cancer (17.8 per 100,000 women) and cause of cancer deaths $(9.8$ per 100,000$)$ among all types of cancer in women [11]. Human papilloma virus plays a central role in the development of cervical neoplasia and can be detected in 99.7 percent of cervical cancers [12].

Based on the results of this study, the most frequent histological type were scuamocellular cancers, an incidence relatively higher than the incidence of this subtype in countries where an active prevention of HPV infection through vaccination is performed [13]. Although we encountered a relatively high percentage of stage I tumors, most cases on diagnosis were 
stage IIB, probably due to the lack of screening programs and the ignorance of the symptoms by the patients. The selection of patients for neoadjuvant treatment was performed accordingly to the general status, comorbidities and the tumoral stage. In stage IIB cases in which neoadjuvant treatment was not performed due to the medical conditions of the patients it was considered to be at high risk of developing a severe postoperative complication due to the summation of radio-chemotherapy and surgery. One major reason for performing primary surgery was acute bleeding which was not controlled through local treatment, either surgical or intracavitary radiotherapy. Patients who undergone neoadjuvant treatment presented significantly lower values of haemoglobin and white blood cells due to the toxicity radio-chemotherapy. Anemia is common among women with cervical cancer and its etiology is often multifactorial: tumour bleeding, poor nutritional status, anemia of chronic disease, and treatment-related myelosuppression. On average, hemoglobin levels can decrease by approximately $0.4 \mathrm{~g} / \mathrm{dL}$ per week during concomitant Cisplatin-based chemoradiotherapy [14]. Based on histological examination we encountered a complete response rate of 16 from 38, similar to the percentages reported in the literature [15]. There were two cases with complete pathological response on tumor, but with positive lymph nodes, this represents a reasonable argument for the surgical treatment after chemoradiation even in patients with a complete clinical response.

The extent and the quality of surgery influences the number of perioperative complications, the appropriateness of adjuvant therapy, overall morbidity, local tumour control and consequently probably survival. The literature supports a positive relationship between number of cases operated on and outcome for different types of cancer, indicating a benefit for centralization of care pathways [16]. Extent of lymphadenectomy is traditionally measured using the number of lymph nodes removed. The EORTC-GCG agreed that the minimum number of pelvic lymph nodes that must be removed is 12 [17].

Urinary tract injury is the most common complication of pelvic surgery. Its incidence is reported to be from 0.5 to $1.5 \%$, and bladder injury is more common than ureter injury [18]. Most bladder injuries, but only one third of all ureter injuries, are identified during surgery. The most common sites of ureteral injury during hysterectomy are along the pelvic wall lateral to the uterine artery, the area of uretero-vesical junction, and the base of infundibulo-pelvic ligament [19]. The timing of repair of ureteric injuries is somewhat controversial, with some urologists advocating immediate repair whereas others favor delayed repair [20].

This study has two major drawbacks; one is the relatively small number of cases included in the analysis. All patients included in this series were treated in a tertiary centre by a specialized team involved and dedicated to the treatment of gynecological malignancies, with relatively similar preoperative management, operative technique and postoperative surveillance. The other one is the heterogeneity in terms of tumoral stages, we preferred to include in the analysis all patients in which surgery was performed, and this gives an insight of the actual status of patients diagnosed with this health problem.

Cervical cancer remains an important health issue in Romania due to the high percentage of advanced cases on diagnosis. Tailoring an appropriate treatment involves a multidisciplinary team highly trained and experienced in management of this disease. Radical hysterectomy is performed as a primary therapy for stages IB or IIA and may be used as salvage therapy in patients with recurrent or persistent cervical cancer limited to the cervix. For patients with locally advanced cervical cancer it is preferred primary chemoradiotherapy, the indication for surgery must be done after a thoroughly reevaluation of the response. Improvements in surgical technique, 
prophylactic antibiotics, thromboembolic prophylaxis and perioperative care have lowered operative morbidity from this procedure.

\section{CONFLICT OF INTEREST}

Authors have no conflict of interest to declare.

\section{REFERENCES}

1. Kesic V, Poljak M, Rogovskaya S. Cervical cancer burden and prevention activities in Europe. Cancer Epidemiol Biomarkers Prev. 2012; 21(9): 1423-1433.

2. Piver MS, Rutledge F, Smith JP. Five classes of extended hysterectomy for women with cervical cancer. Obst. Gynecol. 1974; 44(2): 265-272.

3. Michalas S, Rodolakis A, Voulgaris Z, Vlachos G, Giannakoulis N, Diakomanolis E. Management of early-stage cervical carcinoma by modified (Type II) radical hysterectomy. Gynecologic oncology. 2002; 85(3): 415-422.

4. Landoni F, Maneo A, Cormio G, et al. Class II versus class III radical hysterectomy in stage IB-IIA cervical cancer: a prospective randomized study. Gynecol Oncol. 2001; 80(1): 3-12.

5. Sartori E, Zanagnolo V. [Complications following radical hysterectomy for cervical carcinoma]. Recenti progressi in medicina. 2003; 94(12): 562-567.

6. Ware RA, van Nagell JR. Radical hysterectomy with pelvic lymphadenectomy: indications, technique, and complications. Obstetrics and gynecology international. 2010; 2010. doi: pii: 587610. 10.1155/2010/587610.

7. Charlson ME, Pompei P, Ales KL, MacKenzie CR. A new method of classifying prognostic comorbidity in longitudinal studies: development and validation. Journal of chronic diseases. 1987; 40(5): 373-383.

8. Mota F, Vergote I, Trimbos JB, et al. Classification of radical hysterectomy adopted by the Gynecological Cancer Group of the European Organization for Research and Treatment of Cancer. International journal of gynecological cancer. 2008; 18(5): 11361138.
9. Common Terminology Criteria for Adverse Events (CTCAE) v4.0. National Cancer Institute, [available at http://ctep.cancer.gov/ protocolDevelopment/electronic_applications/ ctc.htm]

10. Pecorelli S, Zigliani L, Odicino F. Revised FIGO staging for carcinoma of the cervix. Int J Gynaecol Obstet. 2009; 105(2): 107-108.

11. Jemal A, Bray F, Center MM, Ferlay J, Ward E, Forman D. Global cancer statistics. $C A$ Cancer J Clin. 2011; 61(2): 69-90.

12. Walboomers JM, Jacobs MV, Manos MM, et al. Human papillomavirus is a necessary cause of invasive cervical cancer worldwide. The Journal of pathology. 1999; 189(1): 12-19.

13. Ries LAG, Melbert D, Krapcho M, et al. SEER Cancer Statistics Review, 1975-2004. National Cancer Institute; Bethesda, MD 2007

14. Kapp KS, Poschauko J, Geyer E, et al. Evaluation of the effect of routine packed red blood cell transfusion in anemic cervix cancer patients treated with radical radiotherapy. Int $J$ Radiat Oncol Biol Phys. 2002; 54(1): 58-66.

15. Classe JM, Rauch P, Rodier JF, et al. Surgery after concurrent chemoradiotherapy and brachytherapy for the treatment of advanced cervical cancer: morbidity and outcome: results of a multicenter study of the GCCLCC (Groupe des Chirurgiens de Centre de Lutte Contre le Cancer). Gynecol Oncol. 2006; 102(3): 523-529.

16. Hillner BE, Smith TJ, Desch CE. Hospital and physician volume or specialization and outcomes in cancer treatment: importance in quality of cancer care. J Clin Oncol. 2000; 18(11): 2327-2340.

17. Benedetti-Panici P, Maneschi F, D'Andrea G, et al. Early cervical carcinoma: the natural history of lymph node involvement redefined on the basis of thorough parametrectomy and giant section study. Cancer. 2000; 88(10): 2267-2274.

18. Aronson MP, Bose TM. Urinary tract injury in pelvic surgery. Clin Obstet Gynecol. 2002; 45(2): 428-438.

19. Liapis A, Bakas P, Giannopoulos V, Creatsas G. Ureteral injuries during gynecological surgery. Int Urogynecol J Pelvic Floor Dysfunct. 2001; 12(6): 391-393.

20. Selzman AA, Spirnak JP. Iatrogenic ureteral injuries: a 20-year experience in treating 165 injuries. J Urol. 1996; 155(3): 878-881. 
